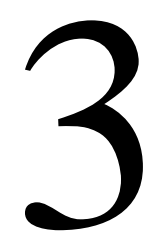

\title{
UN CUENTO DE DOS PAÍSES: EDUCACIÓN A DISTANCIA DE MÉXICO Y ESTADOS UNIDOS DE AMÉRICA
}

\author{
A Tale of Two Countries: Higher Distance Educación \\ in Mexico and the United States of America
}

\author{
Pedro Cookson Steele* y Nancy Domínguez González ${ }^{* *}$
}

\section{$\underline{\text { RESUMEN }}$}

Este artículo presenta un resumen del panorama de la educación superior a distancia en dos países vecinos del norte del continente americano: México y los Estados Unidos de América. La primera sección aborda una descripción de las diferentes formas de distancia ofrecidas por las universidades mexicanas, así como su evolución histórica, los retos que enfrenta en materia de evaluación, acreditación, posicionamiento social como alternativa educativa de calidad y los beneficios que representa para una sociedad en crecimiento. La segunda sección aborda la educación superior a distancia en los Estados Unidos, relata los antecedentes históricos y presenta cuatro tipos de oferta de educación de distancia. Repasamos los resultados de informes nacionales de educación a distancia superior publicados desde 2005 al 2015. Además de comentar algunos indicadores de la madurez del campo profesional de la educación a distancia, discutimos las respuestas de instituciones y asociaciones profesionales al enfrentarse a nuevas reglas emitidas por el Departamento de

\footnotetext{
* Adelante Online (Estados Unidos).

** Universidad Veracruzana (México).
} 
Educación del gobierno federal concernientes a la entrega de programas y cursos de educación a distancia a través de las fronteras estatales.

PALABRAS CLAVE: Universidad a distancia, participación de los estudiantes, calidad de la educación, programas de educación a distancia, política gubernamental.

\begin{abstract}
$\underline{\text { ABSTRACT }}$
This article presents a summary of the panorama of higher distance education in the two neighboring countries of North America of Mexico and the United States. The first part addresses the different forms of distance education offered by Mexican universities - how, as they have evolved, they have faced such challenges as evaluation, accreditation, and served as an alternative route to social mobility, thus benefitting a society undergoing development. The second section addresses higher distance education in the United States, recounting the historical background and the different forms of distance education offer. We review the findings of national studies of higher distance education published from 2005 to 2015. In addition to commenting on some indicators of distance education as a professional field of practice of distance education, we discussed the responses institutions and professional associations have responded to the interpretation of the federal government's Department of Education's rules on delivery of distance education courses and programs across state lines.
\end{abstract}

KEY WORDS: Open University, Participation of Students, Quality of Education Programs of Open University, Government Policy.

$* * * * *$

\title{
1. INTRODUCCIÓN
}

El propósito de este artículo es presentar el panorama de la naturaleza y el alcance de la educación a distancia en dos países vecinos del norte del continente americano: México y los Estados Unidos de América. Se relatarán los antecedentes históricos de la escena actual, las diferentes maneras en que se ofrecen los cursos y programas a distancia y las características de las instituciones a distancia dentro del contexto de los sistemas de educación en la educación superior. Un punto interesante a discutir es el relacionado con la cuestión de calidad de la instrucción a distancia y las reglas propuestas por los gobiernos federales de ambos países para la entrega inter-estatal de los cursos y programas a distancia. Se hará breve mención de algunos aspectos que reflejan la maduración de este campo de práctica. Hemos limitado nuestro enfoque a la educación a distancia en el contexto de las instituciones educativas que ofrecen educación superior de carácter formal, 
por lo que la educación a distancia en otros niveles educativos y de carácter no formal o informal no se analiza en este estudio.

\section{EDUCACIÓN SUPERIOR A DISTANCIA EN MÉXICO}

\subsection{Antecedentes históricos}

La educación a distancia en México tiene una historia de cerca de 100 años, en los que ha pasado por etapas muy variadas y complejas. El primer antecedente de esta modalidad educativa que se tiene data de inicios del siglo XX, cuando en 1923 el gobierno federal a través de la Secretaría de Educación Pública (organismo encargado de la educación en el país) implementó las misiones culturales, las cuales tenían el propósito de ofrecer programas de capacitación a maestros de escuela básica directamente en sus áreas de trabajo. Esta iniciativa partió de la idea de establecer una estrategia para capacitar a maestros de educación básica que se encontraban prestando sus servicios en áreas rurales dispersas a lo largo del territorio nacional, de tal forma que pudieran mejorar su formación sin desatender a sus estudiantes y coadyuvar con ello a reducir los índices de analfabetismo que, a principios del siglo XX, alcanzaban el 95\% de la población y conformar así una nación civilizada. El proyecto funcionó eficientemente y obtuvo la confianza y colaboración de muchos de los docentes, por lo que en 1932 las misiones culturales pasaron a integrarse a las escuelas rurales y se volvieron fijas (HERRERA, 2014). Se considera éste el primer antecedente de la Educación a Distancia en México, porque los estudiantes estaban separados física y temporalmente de la institución que ofertaba la formación y de sus compañeros, más no del facilitador, en este caso los misioneros. Hay quienes sostienen que el primer registro de Educación a Distancia en el país se refiere al modelo implementado por la asociación filantrópica "Compañía Lancasteriana" que data de 1822, cuyo fin era promover la educación primaria entre los sectores marginados de la capital del país (BOSCO, 2008). Sin embargo sigue siendo el caso de las misiones educativas el ejemplo más claro del inicio de la educación a distancia en el país.

A partir de los años 40, los destinatarios de la educación a distancia fueron fundamentalmente los profesores no titulados del campo mexicano, la institución que se responsabilizó de su formación y titulación fue el Instituto Federal de Capacitación del Magisterio (IFCM) con una estrategia basada en materiales impresos y el uso de la radiodifusión. El IFCM dejó de existir en 1971, pero su modelo educativo fue imitado por otras instituciones formadoras de docentes. Fue en 1944 cuando se creó el Instituto Federal de Capacitación del Magisterio, cuyo objetivo era capacitar a los maestros en servicio, y fue entonces que se diseñaron y distribuyeron los primeros cursos institucionales de educación por correspondencia en este país. Se enviaban cuadernillos coleccionables y cuestionarios 
que los estudiantes debían resolver y devolver a la oficina central. La instrucción adquirida se complementaba con cursos intensivos en periodos vacacionales (CENTRO DE ACTUALIZACIÓN DEL MAGISTERIO, 2015). En esa década también empezaron a registrarse avances en tecnología, especialmente relacionados con televisión; además, se fundaron escuelas de enseñanza audiovisual, se desarrollaron proyectos relacionados con la televisión y este medio de comunicación cobró importancia en el panorama nacional. En 1947 se transmitió por primera vez una cirugía por circuito cerrado, lo cual puede constituirse como el primer esfuerzo por transmitir por televisión material con objetivo instruccional (BOSCO, 2008).

Sin embargo, es hasta la década de los 60 que se inicia el uso a gran escala de la televisión educativa con el programa de telesecundarias, mismo que aún existe y a la fecha llega a más de 30,000 sitios, que si bien no es educación superior, ha abierto líneas interesantes de acción e investigación sobre la educación a distancia en este contexto. El uso de la radio también abrió escenarios educativos de importancia como la emisión de cursos de alfabetización, complementados con materiales impresos y la comunicación directa eventual. Otro ejemplo son cursos de inglés por radio que se ofrecían por parte de instituciones educativas públicas como la Universidad Autónoma de Sinaloa.

En los 70's, con el ejemplo de la Open University del Reino Unido, surgen en México programas como el Sistema de Universidad Abierta de la UNAM o el Sistema Abierto de Enseñanza del Instituto Politécnico Nacional. Basado más en materiales impresos que en el uso de medios electrónicos masivos, hacia 1979 se creó el Sistema de Educación a Distancia de la Universidad Pedagógica Nacional (UPN), con el propósito de apoyar la formación de profesores de educación básica. Con la creación de este sistema de Educación a Distancia, la UPN fue la primera institución pública de educación Superior en México que cubrió todo el país con esta modalidad, al establecer unidades en todos los estados de la República. En una primera etapa la estrategia se apoyó básicamente en materiales impresos.

Desde los 80 hasta el presente, la atención se ha centrado en las tecnologías de la información y la comunicación que se insertan reenfocando los modelos de comunicación generados con las aportaciones del pasado; podemos decir que los nuevos medios no llegaron a desplazar a los existentes, sino que vinieron a diversificar el menú de posibilidades, de manera que los discos compactos, la educación vía satélite y los ambientes virtuales de aprendizaje en Internet, conviven con los textos impresos, el uso del teléfono móvil, los dispositivos inteligentes y otros medios en una permanente búsqueda de las mejores opciones para la educación a distancia. Cierto es que los medios anteriores se usan cada vez menos y la comunicación directa se ve desplazada poco a poco por redes sociales y demás herramientas que provee Internet. Los medios más utilizados son en primer lugar todos aquellos suministrados por Internet, posteriormente los impresos, 
enseguida la televisión y muy poco la radio. La correspondencia tradicional y el teléfono tienen básicamente un uso administrativo y se usa escasamente con fines académicos.

En el caso de México los propósitos y modos de organización de la educación a distancia son de lo más variado, debido tanto a la diversidad de objetivos y condiciones institucionales como a las influencias que se han dado en su origen y desarrollo. Entre esas influencias son de destacar la tradición mexicana y la influencia de instituciones europeas, estadounidenses, latinoamericanas y de organismos internacionales como la UNESCO (MORENO, 2009).

En 2014 el sistema educativo nacional registraba a 3.3 millones de estudiantes matriculados en programas de nivel superior, incluyendo postgrado; se espera que esa cifra se verá incrementada para cubrir en el 2020 al 29\% de la población entre 20 y 24 años. En dos escenarios de crecimiento de la matrícula de licenciatura, la Asociación Nacional de Universidades e Institutos de Educación Superior (ANUIES) prevé una atención a la demanda del $29 \%$, en el caso más optimista, y del $26 \%$, en la más moderada, del grupo de edad de 20 a 24 años. Estas proporciones de atención, en cualquiera de los escenarios propuestos, resultan del todo insuficientes frente a los parámetros de atención de los países desarrollados, que de acuerdo con un informe publicado por el Banco Mundial alcanzan un promedio superior al 50\%, mientras que en los países de ingresos medios es del $21 \%$. Esto significa que, aún si comparamos a México con países de nivel de desarrollo similar, la atención en el nivel superior es inferior y definitivamente insuficiente (ANUIES, 2015).

\subsection{Situación actual}

La Constitución Política de los Estados Unidos Mexicanos postula que toda la educación en el país debe estar normada y regulada por la Secretaría de Educación Pública (SEP), aun aquella que se oferta en instituciones privadas, por lo que es esta Secretaría la que otorga los reconocimientos de validez oficial que permiten que un profesional pueda ejercer libremente en el territorio nacional. De tal suerte que un programa educativo para ser reconocido debe cumplir los lineamientos que marca la SEP.

A continuación se presenta una visión general de la educación a distancia en el país, haciendo énfasis en que se presenta información de aquellos programas que cuentan con ese registro de validez oficial. De los programas que se ofrecen en los niveles de licenciatura y postgrado, así como en programas de formación continua en educación abierta y a distancia, se observa que un total de 57 instituciones educativas ofrecen 211 programas de licenciatura y dos programas de Técnico Superior Universitario. En el nivel de postgrado, las mismas instituciones ofrecen un sinnúmero de programas de diplomado y cursos profesionales, 28 especialidades, 175 programas de maestría, y 16 programas doctorales. Se cubren todas las áreas del conocimiento, aunque definitivamente la atención 
está centrada en las ciencias sociales y administrativas, seguidos en importancia por los de ingeniería y tecnología, ciencias agropecuarias y los del área de educación y humanidades; son escasos los programas en ciencias de la salud y los de ciencias exactas. Además de estos programas contabilizados, y que están debidamente acreditados, hay que añadir un sinnúmero de programas que no cuentan con registro de validez oficial debido a que su naturaleza no les permite ser reconocidos por la SEP (medicina alternativa, por ejemplo) o aquellos que están en proceso de obtener dicho registro (EL PORTAL DE LOS ESTUDIANTES, 2015).

En importante destacar que muchas universidades públicas han hecho esfuerzos importantes por ampliar su oferta en programas educativos a distancia, sin embargo al momento la mayor oferta de estos programas se encuentra en universidades privadas. Se calcula que a finales del 2012 ambos tipos de instituciones públicas y privadas atendieron a un total de 260.000 estudiantes, tanto en nivel de técnico superior universitario, licenciatura, maestría y doctorado. Esto representa un número alto si se compara con los 15.000 estudiantes que se atendían en 1997, sin embargo es bajo en proporción si se le compara con el crecimiento que otros países han presentado en esta modalidad educativa, y sobre todo con la demanda de la propia sociedad de educación de nivel superior, por lo que la cifra resulta insuficiente (EL UNIVERSAL, 2012).

En relación con la infraestructura necesaria para los programas a distancia, los esfuerzos han sido diversos. En primer lugar puede señalarse aquel realizado por el Instituto Latinoamericano de la Comunicación Educativa (ILCE) y la Red EDUSAT que posibilita la recepción de la señal en 30 mil puntos, entre los cuales hay un número importante de instituciones educativas. Por otra parte, se encuentra la Red Nacional de Videoconferencia, en la que se cuenta con 120 salas aproximadamente; la Red del Noroeste y además un conjunto importante de instituciones cuentan con equipo que les permite operar programas a distancia. Las tecnologías de información y comunicación de las que se dispone hoy en día, posibilitan que quienes no tengan acceso a una red de videoconferencias puedan establecer contacto con las personas que forman su grupo de estudio a través de una computadora con conexión a Internet, con un nivel de eficiencia cada vez más alto. En las instituciones educativas se ha convertido en un asunto de atención constante la actualización de equipo de cómputo, software y acceso a Internet, contratación de bases y bancos de datos remotos. Lo anterior significa que, con diferentes grados de avances, en un importante conjunto de instituciones educativas se cuenta con la infraestructura suficiente para operar programas a distancia.

En este punto, es importante destacar una iniciativa que surgió en México en 2013 que se denomina "Internet libre para todos" (INTERNET PARA TODOS, 2013), que mediante un movimiento social busca que se garantice mediante la ley que toda la población en el país pueda acceder a Internet desde cualquier punto: tanto edificios y 
espacios públicos como privados; para hacer valer en México el derecho humano de contar con acceso a la vía de comunicación más importante del mundo. Para ello, se proponen acciones como emplear la infraestructura de la Comisión Federal de Electricidad para establecer los nodos de conexión, que se abran los mercados de oferta del servicio, liberar los espacios blancos que reserva la televisión analógica y que pronto serán innecesarios, establecer una coalición que presente y dé seguimiento a la propuesta y aglutinar la opinión pública en esta iniciativa.

Una estructura que ha operado con éxito en otros países dentro de la educación a distancia es el consorcio, que es una agrupación de instituciones públicas y privadas unidas en el propósito de ofrecer educación a distancia, generalmente en una región o estado para ayudar a abatir el rezago educativo al atender a toda la población que lo solicita y que no puede ingresar a la oferta con que cuentan las instituciones de manera individual. Un ejemplo de este tipo de organización es el Consorcio Clavijero que se constituyó en el estado de Veracruz en 2005 y desde entonces ha evolucionado como un organismo que contribuye en abatir el rezago educativo en México específicamente en el territorio veracruzano, mediante la oferta de programas académicos orientados a las áreas estratégicas del conocimiento. El Consorcio actualmente ofrece programa de bachillerato en línea, Técnico Superior Universitario, Licenciaturas y programas de formación continua (INSTITUTO CONSORCIO CLAVIJERO, 2015).

Parte importante de la consolidación de la educación a distancia en el país fue la fundación de la Universidad Da Vinci en 2004, la primera universidad 100\% virtual del país, cuyo objetivo es ofrecer una alternativa competitiva en calidad, costos, tiempos y posibilidad de acceso, en la que se puede obtener formación a nivel profesional y postgrado con un modelo completamente a distancia y mediante la aplicación de las tecnologías de la información y la comunicación de vanguardia, al momento ofrece programas de licenciatura, especialidad, maestrías y doctorados. A partir de ese año se han fundado otras instituciones con el mismo objetivo y con métodos de trabajo variados, que van desde procesos educativos completamente a distancia hasta programas mixtos que requieren asistencia presencial en una $\mathrm{u}$ otra medida.

\subsection{Sobre la acreditación}

Como se mencionó anteriormente, la Secretaría de Educación Pública (SEP) es la encargada de otorgar el registro de validez oficial a los programas educativos de grado y postgrado, sin importar la modalidad en que se oferten. Sin embargo todo programa educativo a distancia en instituciones bimodales ha estado inmerso en un ambiente de institución presencial, por lo que los requisitos para obtener el registro eran, más bien, facilitados por la institución en sus programas presenciales. Es hasta el surgimiento de la primera institución completamente en línea que la SEP se vio en la necesidad de 
reestructurar su lista de requisitos para la obtención del registro, considerando aquellos aspectos que atañen sólo a la educación a distancia. Por ejemplo no es importante que la institución no tenga una biblioteca física, aulas o cafetería, pero es vital que haya una fuerte infraestructura tecnológica que soporte el campus virtual y el sistema de administración de conocimiento elegido, así como un sistema de soporte técnico 24/7, es decir 24 horas al día los siete días de la semana.

Acreditar la calidad de los programas educativos en México es un proceso complejo y hay varias instancias que operan a manera de niveles y que determinan en uno u otro aspecto la calidad del programa de educación superior. Se ha establecido que aquel programa educativo que desee acreditar su calidad académica primeramente debe pasar por un proceso de evaluación ante los Comités Interinstitucionales para la Evaluación de la Educación Superior (CIEES), la cual es una asociación civil que está constituida por pares académicos en diferentes disciplinas que se conforman en comités con la competencia para realizar el proceso de evaluación.

Dicho proceso inicia cuando un programa desea evaluarse. Para ello debe autoevaluarse mediante un instrumento con indicadores que ayudan a identificar si el programa cuenta con los elementos necesarios para certificar su calidad. De no ser así es necesario realizar los ajustes pertinentes, en cuanto se cubren se pide la evaluación a los CIEES, quienes conforman un grupo evaluador con pares de distintas universidades pero de la misma disciplina. Los CIEES, una vez realizado el proceso de evaluación, pueden certificar que el programa es de alta calidad académica (nivel 1) que es un programa que requiere atención en muchos aspectos (nivel 2) o bien que es un programa que conviene eliminar o bien requiere grandes cambios para subsistir (nivel 3).

El dictamen otorgado por los CIEES habilita al programa educativo para pasar al siguiente nivel de evaluación, que en el caso de licenciaturas son los organismos acreditadores, donde también se conforman grupos de expertos en las diferentes áreas del conocimiento designados por el Consejo para la Acreditación de la Educación Superior, A.C. (COPAES), los cuales tienen atribución oficial para determinar si el programa puede ser acreditado en su área con el más alto estándar de calidad en México y toma en cuenta también estándares internacionales. En el caso de la educación a distancia, los CIEES a poco tiempo de su creación en 1991, iniciaron la construcción de un instrumento especializado en evaluar programas educativos en modalidad mixta o a distancia, para lo cual incluyeron un indicador especial que mide parámetros que sólo atañen a la educación a distancia como infraestructura tecnológica, servicio de atención a usuarios tanto académica como soporte técnico, métodos de evaluación adecuados, etc.

En el caso de los programas de postgrado, se certifican por medio de los CIEES, mas no son acreditados por ningún comité del COPAES. Por el contrario es el Consejo 
Nacional de Ciencia y Tecnología (CONACYT) quien determina si un programa es de calidad o no, para lo cual cuenta con un proceso de evaluación que, de cumplirse, permite al programa ingresar al Padrón Nacional de Postgrados de Calidad (PNPC), otorgando un registro que debe ser revalidado en periodos de 3 años. Antes del año 2012 el CONACYT sólo consideraba programas educativos de postgrado presenciales para otorgar su aval de calidad. Para el caso de la educación a distancia, a finales del año 2011 el Conacyt liberó un instrumento de evaluación que permitió por primera vez a inicios de 2012 validar la calidad de los programas de postgrado para ingresar en el PNPC. Aún cuando en ese momento algunos programas de postgrado a distancia lograron su ingreso al padrón, aun se tienen muchos puntos que atender, porque el CONACYT otorgó el aval a los postgrados a distancia de calidad, sin embargo no otorga becas ni ningún tipo de apoyo o estímulo a quienes deciden optar por una maestría o doctorado en línea, basándose para ello en la idea de que no son estudiantes dedicados al $100 \%$ y por ello no requieren manutención o apoyo.

\subsection{Prospectiva}

Se considera que el avance de la educación a distancia en México ha sido lento, pues a la fecha es considerada por muchas personas como educación de baja calidad. Cierto es que ha crecido con serias limitaciones de infraestructura, planeación y recursos; en muchos de los casos por imitación de otros modelos y no con base en estudios de necesidades reales. En este sentido, ayuda la creación de instituciones que se forman con el propósito de aglutinar esfuerzos y realizar investigación que permite generar modelos y parámetros de crecimiento de la modalidad educativa, como el Sistema Nacional de Educación a Distancia, la Universidad Abierta y a Distancia de México, el Espacio Común de Educación Superior a Distancia con su red de investigadores consolidada gracias a la colaboración de universidades que han abierto sus programas en esta modalidad, y como ellas hay otras instituciones que aportan en este sentido.

Uno de los proyectos más recientes en México parte de la Subsecretaría de Educación Superior en coordinación con el gobierno de Francia y consiste en generar comunidades académicas denominadas Comunidades Digitales para el Aprendizaje en la Educación Superior (CODADES) donde participan universidades públicas en la generación de recursos para el aprendizaje en temáticas como:

- Educación.

- Artes, Humanidades y Ciencias Sociales.

- Ciencias Económico-Administrativas.

- Ciencias Naturales y ciencias exactas.

- Ingeniería y tecnología.

- Ciencias agronómicas, y medicina veterinaria y zootecnia.

- Salud. 
- Relaciones internacionales.

En cada una de estas áreas lidera el proyecto una universidad pública reconocida por sus logros en el dicha área, la cual invita a colaborar a pares expertos de otras instituciones para desarrollar en colaborativo contenidos como MOOC's (cursos masivos en línea) objetos de aprendizaje y repositorios de recursos; dichos recursos tienen la función de fortalecer procesos educativos en la educación superior presencial, pero también abrir el panorama sobre la educación a distancia, de tal suerte que la comunidad académica en el país reconozca y se beneficie de las posibilidades de esta modalidad educativa.

A pesar de las dificultades del crecimiento en México de la educación a distancia, cada día cobra fuerza y se constituye en una alternativa viable y de calidad para muchas personas que se encuentran inmersas en dinámicas laborales, familiares y sociales que les impiden acceder a la educación convencional; los avances en las tecnologías de la información y la comunicación junto con la ampliación en infraestructura tecnológica en el país ayudan en este propósito, de tal suerte que cada día se cuentan con más posibilidades de mejorar la educación y mejorar con ello el desempeño laboral en beneficio personal y del entorno en general.

\section{EDUCACIÓN SUPERIOR A DISTANCIA DE LOS ESTADOS UNIDOS}

\subsection{Antecedentes históricos}

Durante los últimos 150 años, el proceso de la educación a distancia comenzaba con el reparto de materiales didácticos impresos mandados a los estudiantes por el sistema postal y la devolución de las tareas escritas por los estudiantes. Este método de la educación a distancia por correspondencia ha ofrecido oportunidades para que los hombres y mujeres a través de actividades de enseñanza-aprendizaje a distancia, facilitadas por la tecnología de información y comunicación, procuren aumentar su conocimiento, destreza y/o sensibilidad para ayudarse a sí mismos, a otras personas, o bien a sus organizaciones. Para evitar la tendencia de alabar la versión corriente de la educación a distancia como algo completamente nuevo e innovador surgido sin precedentes, mantenemos la perspectiva que toda configuración actual de cursos y programas a distancia llevan en pos de sí varios antecedentes. Aquellos antecedentes los trazaremos como parte de una trayectoria histórica que comprende cinco generaciones, las cuales van acompañadas del desarrollo de diferentes tecnologías.

\subsubsection{La primera generación de educación a distancia por correspondencia}

La primera generación de la educación a distancia la enfocamos a los programas de instrucción por correspondencia y el estudio independiente que se desarrolló en el siglo 
XIX, donde se utilizaban materiales didácticos impresos mandados a los estudiantes mediante el sistema postal. Anna Eliot Ticknor, una autora y educadora radicada en Boston, Massachusetts, estableció la primera escuela por correspondencia en los Estados Unidos. "La Sociedad para Alentar los Estudios en Casa", tenía el propósito de "inducir entre las amas de casa el hábito de dedicar parte de cada día al estudio sistemático y exhaustivo". El proyecto funcionó durante un periodo de 24 años; enviaba materiales de estudio por correo a un total de 10,000 miembros, tanto a mujeres de poca educación al igual que mujeres de la clase alta (SOCIEDAD PARA ALENTAR LOS ESTUDIOS EN CASA, 1886). Otro ejemplo de los primeros años de esta primera generación que ofrecía cursos por correspondencia, fue el Instituto Chautauqua que creó The Chautauqua Literary and Scientific Circle, el cual ofrecía un curso de cuatro años por correspondencia para ayudar a los adultos que no tenían ni el tiempo ni el dinero para asistir a una universidad a adquirir destrezas y conocimientos universitarios. Posteriormente, desde 1883 a 1891 el estado de Nueva York autorizó el Chautauqua College of Liberal Arts a conceder grados académicos a estudiantes que completaron sus estudios por correspondencia y participaron en estancias presenciales de verano (NASSEH, 1997). Uno de los que enseñaban cursos de hebreo por correspondencia en Chautauqua fue William Rainey Harper. Cuando éste posteriormente fue nombrado rector fundador de la Universidad de Chicago, creó la División de Extensión, que ofrecía cursos por correspondencia para promover oportunidades educativas para adultos. Otras universidades siguieron el ejemplo y la educación a distancia por correspondencia llegó a ser la versión más popular de la educación a distancia conducida por las instituciones de educación superior desde el fin del siglo diecinueve hasta los años 1960s.

Edelson y Pittman (2001) postularon que esta generación se desarrolló en dos líneas paralelas. Por una línea se desarrolló la educación superior, por la otra se multiplicó en el sector privado. Uno de los ejemplos destacados del sector privado fue el curso sobre seguridad de minería que empezó Thomas J. Foster, que publicaba un periódico sobre la minería en Scranton, Pennsylvania. Foster inició el primero de estos "estudios en casa" con el tema de la seguridad en la minería para los mineros de carbón. Se multiplicaron los cursos por correspondencia en la década de los 1880 y llegaron a ser la International Correspondence Schools, la escuela privada por correspondencia más grande del mundo. En 1894 matriculó 2,500 personas, en 1895 matriculó 72,000 y en 1906, unos 900.000 (WATKINS, 1996). En lugar de lecciones individuales, a los estudiantes se les enviaban por correo libros de texto completos. Se diferenciaba también por contar con "1200 agresivos vendedores" (CLARK, 1906). En vez de calificar a los estudiantes para una variedad de opciones de empleo generales, los cursos prepararon a los estudiantes para desempeñar únicamente destrezas de empleo prácticas y específicas (MOORE y KEARSLEY, 2005). 
Al proliferar la popularidad de educación a distancia por correspondencia, algunas escuelas en el sector privado empleaban prácticas engañosas que afectaron la reputación de la educación a distancia. Algunas de estas instituciones inscribían a muchos estudiantes a quienes cobraron las cuotas al comenzar los cursos, sabiendo de antemano no tendría altos costos atenderles cuando abandonaran sus cursos. Como legado de estas prácticas, en la década de los 50, el gobierno federal decretó reglas que limitaban y luego retiró su apoyo financiero a los estudiantes que estudiaban por este medio. Desde entonces, el tema de la baja calidad de la educación a distancia instada por esas escuelas en esta primera generación ha permanecido vivo (SABA, 2011). A pesar de estas prácticas de algunas instituciones en el sector privado, los programas a distancia tuvieron buena acogida entre los proveedores de formación profesional, los servicios militares, y las empresas y universidades, que siguieron ofreciendo cursos y programas mediante el estudio independiente hasta mediados de los 60 del pasado siglo.

\subsubsection{La segunda generación de radio y televisión}

La segunda generación se inició con el desarrollo de las tecnologías de la radio y la televisión, tecnologías que fueron utilizadas para distribuir programación educativa a estudiantes que, por incompatibilidad de horario o geografía, les habría resultado difícil asistir a instituciones educativas ligadas a lugares y horarios fijos. Según Saba (2011, p. 12), el gobierno federal otorgó la primera licencia para establecer una estación de radio educativa a la Universidad de la Iglesia de Jesucristo de los Santos de los Últimos Días en la ciudad de Salt Lake City, Utah en 1921. El año siguiente, en 1922, las Universidades de Wisconsin y de Minnesota recibieron licencias para establecer estaciones de radio educativas (SAETTLER, 1990). Desde entonces las instituciones educativas han continuado operando estaciones de radio. Recientemente, en abril de 2014, la Comisión Federal de Comunicaciones emitió licencias con el mismo propósito a 4,057 estaciones educativas (así llamadas) de frecuencia modulada (cuyos canales ocupan la banda menor (87.1 $91.9 \mathrm{MHz}$ ). En vez de emitir programación educativa, algunas de las estaciones de baja potencia con un rango de cinco o seis kilómetros transmiten noticias, programas culturales y de servicio a la comunidad.

En cuanto al medio de la televisión, Iowa State University fue la primera institución educativa que solicitó licencia para emitir programas educativos por televisión en 1945 y la inició a operar en 1950 (SAETTLER, 1990). A partir de ese año muchas universidades de otros estados también decidieron operar programas de extensión educativa y difusión cultural a través de televisión. Numerosos gobiernos estatales en los Estados Unidos también establecieron canales de televisión educativa, aunque las expectativas siempre excedieron el número real de personas que tomaron ventaja de dichos programas.

\subsubsection{La tercera generación de sistemas de teleconferencias}


La tercera generación de educación a distancia abarcó los sistemas de teleconferencia que debe su prominencia por basarse en los multimedios que incorporaron audioconferencias, videoconferencias y enseñanza asistida por ordenador. Como ejemplo de "teleconferencia", cuando uno de los autores de este artículo dirigió el programa de postgrado de educación permanente en la Universidad Estatal de Pennsylvania, fue pionero en la entrega de cursos de postgrado por videoconferencia en 1985. Cuando limitaciones de presupuesto nos obligaron a utilizar la tecnología menos costosa de las audioconferencias, nos fue posible ofrecer nuestros cursos no sólo a tres recintos universitarios en Pennsylvania, sino también en 1995 a cinco centros de estudios en Finlandia, dos en Estonia y tres en México. Aparte de las reuniones semanales a través de audioconferencias, los instructores interactuaron con personas de contacto en los distintos grupos de estudiantes por medio del ordenador.

\subsubsection{La cuarta generación de educación a distancia en línea}

La cuarta generación se basa en los programas en línea transmitidos por tecnologías compuestas por el ordenador personal, redes de Internet, bases de datos, conversaciones asincrónicas o sincrónicas, acceso a bases de datos y a bibliotecas digitales. A través de sistemas de gestión de aprendizaje, esta generación incorpora aspectos de generaciones previas. Una variación de esta generación vigente, la educación semipresencial (blended o hybrid en inglés), combina la instrucción en línea con la instrucción presencial. Algunos educadores afirman que la interacción cara a cara maestro-estudiante in situ agregadas a las sesiones, resulta en un aprendizaje superior a la educación puramente en línea. Por cierto, tales afirmaciones tendrán sentido en situaciones de "flipped classrooms" (aulas invertidas). En éstas los estudiantes que asisten a cursos presenciales también miran en línea conferencias grabadas para aprender el contenido y después se reúnen físicamente en el campus con sus profesores y otros estudiantes para realizar actividades y tareas de aplicación, participar en discusiones y resolver preguntas. Sin embargo, demandar que los estudiantes ubicados lejos de un campus que concurran a aulas lejanas, resultaría en levantar la misma barrera que deseaban evitar cuando decidieron a estudiar a distancia.

\subsubsection{La quinta generación de educación a distancia por medio de Internet}

Además de contar con los mismos elementos tecnológicos de la cuarta generación, la quinta generación se aprovecha del uso de las capacidades asociativas e interactivas de Internet. Es decir, los estudiantes comparten con sus profesores la responsabilidad de fijar los objetivos, estructura, y contenidos de lo aprendido. Al igual que en la generación anterior, hay proponentes de la educación semipresencial que desean combinar los componentes a distancia por Internet con instrucción presencial dentro de aulas.

\subsection{Distintos tipos de oferta de la educación a distancia}


La mayoría de los cursos y programas a distancia que las instituciones de la educación superior presentan hoy en día reflejan la cuarta y quinta generación antes descritas. Empero, la concesión del reconocimiento y aceptación del avance académico de los estudiantes a distancia por su carrera académica, comprende diferencias significativas entre las distintas instituciones, las cuales consideramos como cuatro categorías diferentes de oferta de educación a distancia. En vez de enfocar, como lo hizo Verduin (1991), en distintos tipos de organizaciones de educación a distancia al nivel post-secundaria, nos enfocamos en cuatro diferentes categorías de oferta a distancia. A continuación describimos estas cuatro categorías de oferta.

\subsubsection{Tipo 1: Instituciones Monomodales de Reconocimiento de Competencias}

Para estudiantes separados temporal o geográficamente de las instituciones en las cuales están inscritos. Esta primera categoría de oferta comprende la práctica de reconocer competencias en vez de créditos basados en la finalización de cursos. Les proporciona a los estudiantes oportunidades para demostrar competencias logradas mediante exámenes, portafolios, competencias documentadas, certificados, licencias, registros de capacitación en el servicio militar u otras experiencias de empleo o trabajo. A continuamos presentamos tres instituciones que ejemplifican esta primera categoría de oferta:

Western Governors University (la Universidad de los Gobernadores del Oeste), ubicada en Salt Lake City, Utah, fue fundada por los gobernadores de los estados de Utah y Colorado en 1965 y empezó a operar en 1967. En lugar de crear su propio contenido de aprendizaje (y así depender del diseño y oferta de cursos), WGU se aprovecha de los recursos de terceros que utilizan tecnología para "ofrecer enseñanza de calidad", así disminuye los costos y permite el aprendizaje asincrónico" (MENDENHALL, 2012, p. 117). Para desagregar las funciones de los profesores, la WGU emplea especialistas que fungen como expertos de contenido y otros que fungen como mentores. Éstos trabajan con los estudiantes individualmente para darles asesoramiento, orientación, motivación, ayuda en línea para identificar los materiales de aprendizaje, medición de competencias y trabajo logradas. No se permite aprobar a los estudiantes hasta que estos demuestren que han logrado las competencias identificadas.

Una de las características distintivas de WGU es que "usa la tecnología con el propósito de proporcionar la educación a distancia que es accesible, flexible y económica, sin poner en peligro la calidad" (MENDENHALL, 2012, p. 117). Por eso la universidad representa una opción asequible para los estudiantes. La matrícula por año de doce meses en 2014-15 fue US\$6,070 por cuantas materias se deseen estudiar. Entre los años 2010-15, este costo se incrementó $0.1 \%$, en contraste el incremento anual promedio de las universidades públicas de cuatro años durante el período 2004-15 fue 2.8\% (COLLEGE BOARD, 2015). Aun así WGU como unidad privada sin fines de lucro, se ha probado que 
es autosuficiente, rentable y no depende de financiamiento por parte del gobierno de ningún estado donde ofrece sus programas. Otro punto que contrasta es que en vez de medir el progreso académico de los estudiantes en términos de la cantidad de tiempo y las notas recibidas al completar cursos, como lo hacen las universidades presenciales, la WGU mide el progreso de los estudiantes al medir las competencias logradas. El resultado de estas diferencias es un incremento en la productividad de los estudiantes. En lugar de los 48 meses que llevan los estudiantes en los programas presenciales en las instituciones tradicionales, el tiempo promedio que llevan los estudiantes para obtener el grado de la licenciatura es 30 meses.

Thomas Edison State College, una institución pública del estado de New Jersey, otorga créditos a los estudiantes a distancia, ofrece para sus cursos a distancia estudios guiados, paquetes de materiales para estudios autodidácticos y exámenes integrados vigilados. Se enfoca en adultos autodirigidos; su misión es proveer oportunidades de aprendizaje post-secundario flexible y de alta calidad. Los estudiantes generan créditos por comprobación de lo que ya saben por medio de su formación profesional, capacitación militar u obtención de licencias y certificados, evaluación de portafolios compuestos de materiales que demuestran conocimientos y destrezas aprendidos y transferencia de créditos concedidos por otras instituciones. Esa institución es un líder reconocido en el uso de las tecnologías educativas y en la valoración de aprendizaje de adultos.

Empire State College, una universidad pública patrocinada por el estado de Nueva York, otorga hasta tres-cuartos de sus grados de licenciatura con base a créditos transferidos de otras instituciones, estudios independientes, evaluación de aprendizaje previo, actividades por grupos de estudio, cursos en línea y semipresenciales (BENKE, DAVIS, y TRAVERS, 2012). Sus valores medulares son formados por un modelo mediante el cual un mentor trabaja individualmente con estudiantes para que definan sus propias necesidades y metas de carrera académica.

\subsubsection{Tipo 2: Instituciones Monomodales Enfocadas en la Oferta de Cursos}

La segunda categoría que comprende la oferta a distancia se integra por las instituciones postsecundarias monomodales, las cuales ofrecen cursos y programas llevados por tecnologías a estudiantes de los que están separados por tiempo y geografía. A diferencia de las instituciones que conforman la primera categoría de oferta, éstas reconocen el avance académico de sus estudiantes en la medida que completen sus programas de estudio en línea. En lugar de requerir que los estudiantes asistan físicamente a clases presenciales en un campus, permiten que se aprovechen de la tecnología para avanzar su carrera académica. Unos cinco ejemplos notables de instituciones privadas monomodales se presentan a continuación: Capella University (http://www.capella.edu/about/), Walden University (http://info.waldenu.edu/walden- 
programs/), American Public University (http://www.apus.edu/, y Jones International University http://www.jiu.edu/explore/online.php.

\subsubsection{Tipo 3: Oferta de Educación a Distancia por Universidades de Modo Doble}

La tercera categoría de oferta de la educación a distancia es la que emprenden la mayoría de instituciones de modo dual, cuya primera prioridad es atender a los estudiantes presenciales reunidos en aulas ubicadas en recintos de "ladrillo y mortero." Como prioridad adicional tales instituciones ven conveniente crear cursos y programas en línea para atender a estudiantes remotos. A menudo, este enfoque secundario se encarga a una división especializada en la entrega de cursos, programas y/o grados a distancia a través de Internet. A veces por razones de conveniencia de horario, sus estudiantes presenciales optan también por estos cursos a distancia. Algunos ejemplos de instituciones privadas y públicas comprendidas en esta categoría de oferta incluyen: Brigham Young University-Idaho (http://byui.edu/online/degrees-and-programs), Rio Salado College Online (http://www.riosalado.edu/Programs/Pages/default.aspx), University of Arizona (http://uaonline.arizona.edu/programs), University of Maryland University College Online (http://www.umuc.edu/) y World Campus de Pennsylvania State University (http://www.worldcampus.psu.edu/).

No todas las universidades que ofrecen cursos a distancia han procedido a establecer programas de grados completos usando las tecnologías educativas. Por ejemplo, estas tres universidades privadas ofrecen cursos a distancia pero no ofertan programas educativos completos a distancia: Harvard University (http://www.extension.harvard.edu/degreesprograms?gclid=CjwKEAiAo8emBRCX_OqU4qek6y8SJACzgf8fQTEDE73o1t1O7ytzeh AMPT6apIQ1PSpegZxl-PVm0RoCnJDw_wcB), University of Phoenix (http://www.phoenix.edu/degrees.html) y Yale University (http://online.yale.edu/).

\subsubsection{Tipo 4: Oferta de Cursos Abiertos Masivos en Línea}

La cuarta generación de oferta de la educación a distancia comprende los cursos abiertos masivos en línea (MOOCs por el acrónimo en inglés de mass open online courses). El término se acuñó en Canadá en 2008 para describir un curso abierto en línea de la Universidad de Manitoba enseñado por George Siemens y Stephen Downes a 25 estudiantes en el campus que pagaron los honorarios para participar presencialmente en el campus y a 2,300 personas del público que se unieron a la clase en línea gratuitamente (DANIEL, 2012).

Desde 2008 esta tipología ha ido evolucionándose y desarrollándose. A pesar de que los MOOCs ya se hayan diseminado a más de 190 países del mundo, ha surtido más efecto en los Estados Unidos. A principios de 2011, Massachusetts Institute of Technology 
estableció una organización para producir MOOCs que llegó a ser edX a la cual se unieron las Universidades de Harvard y de California en Berkeley. En 2012 Stanford University ofreció un MOOC sobre "Inteligencia Artificial" en el cual matricularon 58,000 personas. Uno de los profesores involucrados, Sebastian Thrun, procedió a establecer Udacity como una empresa comercial para ayudar a otras universidades a ofrecer MOOCs. Otros dos profesores de ciencias de computación en Stanford University establecieron Coursera, una organización para ofrecer MOOCs con fines de lucro. Cuando éste ofreció su primer curso, matriculó a 1.4 millones de estudiantes. Resumiendo la importancia estratégica de los MOOCs, Selingo (2014) sustentó que los MOOCs constituían

una fuerza revolucionaria que perturbaría la educación superior tradicional por ampliar el acceso y reducir los costos. La esperanza era que MOOCs -clases ofrecidas por universidades de élite, la mayoría de ellas gratuitas, en algunos casos con cientos de miles de estudiantes cada una- haría posible que cualquiera pueda adquirir una educación, sea un aldeano en Turquía o un abandono de educación post secundaria en los Estados Unidos.

En cuanto a los motivos por agregar este tipo de aprendizaje en línea, éstas fueron las respuestas en la encuesta del Grupo Babson (ALLEN y SEAMAN, 2014, p. 38):

- Incrementar la visibilidad de la institución (26,6\%).

- Experimentar pedagogía innovadora $(18,7 \%)$.

- Impulsar reclutamiento $(17,8 \%)$.

- Oportunidades de aprendizaje flexible (13,5\%).

- Complementar el aprendizaje en el campus $(4,9 \%)$.

- Llegar a servir a nuevos estudiantes $(4,8 \%)$.

- Explorar disminución de costos $(2,4 \%)$.

En cuanto a los métodos que se emplean en los MOOCs, Luhan Mora (2013) explica que

es un curso en línea dirigido de participación interactiva a gran escala y acceso abierto a través del Web. Además de materiales tradicionales, tales como videos, lecturas y conjuntos de problemas, los MOOCs proporcionan foros interactivos de usuarios que ayudan a construir comunidades para los estudiantes, profesores y asistentes de enseñanza... Los MOOCs integran las redes sociales, recursos en línea accesibles y son facilitados por los profesionales principales en el campo de estudio. Más significativamente, los MOOCs se fundamentan en la participación de los estudiantes que auto organizan su aprendizaje de acuerdo con sus propias necesidades de aprendizaje y conocimiento.

Según Daniel (2012), otras características comunes de los MOOCs constan de conferencias por video puntuadas cada 15 minutos con preguntas dirigidas a los estudiantes y materiales de estudio que se acceden a bajo costo o a ningún costo. A pesar de la tasa alta de deserción, gran número de estudiantes matriculados siguen siendo atraídos a los MOOCs. Al evolucionar su modelo de negocio, ya están innovando la entrega no solamente 
con instituciones de la educación superior, sino también con empresas dispuestas de proveer a sus empleados con acceso al contenido de las organizaciones que ofrecen los MOOCs.

Basado en sus dos encuestas nacionales más recientes sobre la educación a distancia, el Grupo Babson (ALLEN y SEAMAN, 2014 y 2015) produjo una imagen actualizada de este tipo de educación en línea. En contraste con algunas predicciones exageradas de los creadores de los MOOCs, que indicaban que reemplazarán la educación superior tradicional enfocada en la instrucción presencial en aulas ubicadas en campus de "ladrillos y mortero", hasta ahora son pocas las instituciones que los han adoptado. En 2012, 2,6\% conducía MOOCs, en 2013, el porcentaje incrementó a 5\%, y en 2014, hasta $8 \%$. En cambio, el porcentaje de instituciones que planeaban ofrecerlos fue un poquito más de $9 \%$ en 2012 y 2013 y descendió a 8\% en 2014. Además el porcentaje de las instituciones que anunciaron ninguna intención de ofrecerlos incrementó desde 33,7\% en 2012, 33.0\% en 2013 , hasta $46,5 \%$ en 2014.

\subsection{Datos sobre el Panorama de la Educación Superior a Distancia}

Entre los años escolares 2002-03 y 2013-14, el Grupo Babson, con el apoyo financiero de la Fundación Sloan-C, condujo encuestas nacionales de los administradores de cabeza de una muestra al azar de las instituciones de la educación superior (ALLEN y SEAMAN, 2002-2003, 2004, 2005, 2006, 2007, 2008, 2009, 2010, 2011, 2012, 2013, 2014, 2015). En todos sus estudios, Allen y Seaman mantenían las siguientes definiciones:

Un curso en línea se define como uno en que al menos el $80 \%$ del curso se entrega contenido en línea. Los cursos presenciales incluyen cursos en que se entregan entre cero y $29 \%$ de los contenidos en línea; esta categoría incluye cursos tradicionales que pueden ser a la vez facilitados por el Web. Los cursos semipresenciales (mixtos o híbridos) son cursos en los cuales entre $30 \%$ a $80 \%$ del contenido del curso está impartido en línea (2015, p. 7).

Basados en estas definiciones, Allen y Seaman compilaron una crónica del desarrollo de la educación a distancia, destacando tales datos como las tasas de crecimiento, percepciones, planes concernientes a los cursos semipresenciales y los completamente en línea. Al revisar todos estos 12 informes del Grupo Babson, quizás el dato más destacado sea el patrón del crecimiento explosivo de las inscripciones en cursos y programas de la educación a distancia en relación a la tasa de crecimiento de todas las inscripciones en toda la educación superior. Como se muestra en la Tabla 1, las tasas de crecimiento de las inscripciones en línea en uno o más cursos han excedido las tasas de crecimiento de la matrícula de educación superior general. En el otoño de 2002, 1,6 millones de estudiantes en línea representaban poco menos del $10 \%$ de las inscripciones en total. Para el otoño del 2012, los estudiantes en línea, 7,1 millones, representaban el 33,5\% de las inscripciones total de educación superior (2013, p. 15). Aunque desde 2011 hasta 2012 la tasa de 
crecimiento de las inscripciones en línea disminuyó a 6,1\%, superó la tasa de crecimiento de 1,2\% de todas las inscripciones para toda la educación superior (2013, p. 15). Cuando el Grupo Babson hizo las dos encuestas recientes (ALLEN y SEAMAN, 2014 y 2015) colaboró para manejar distintos métodos para recoger los datos de todas las instituciones de educación superior con el Sistema de Datos Integrados de Educación Postsecundaria (IPEDS por sus siglas en inglés) del Instituto de Ciencias de Educación del Departamento de Educación (del gobierno federal). Por eso ya no recogieron datos comparables para actualizar la información para los años 2013-14 y 2014-15.

\section{Tabla 1}

Los patrones de crecimiento de la inscripción en general y la inscripción en línea en la educación superior de los Estados Unidos.*

\begin{tabular}{|c|c|c|c|c|c|c|}
\hline 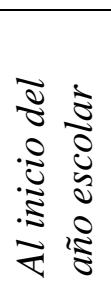 & 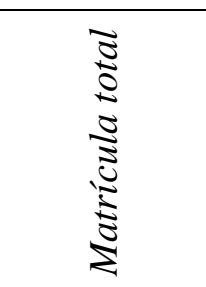 & 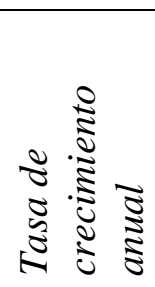 & 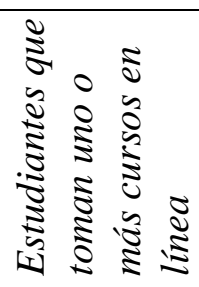 & 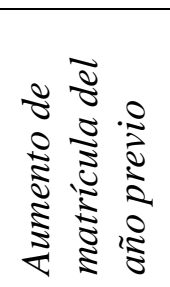 & 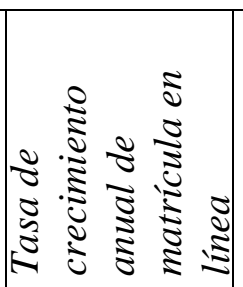 & 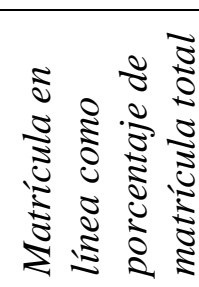 \\
\hline 2002 & 16.611 .710 & NA & 1.602 .970 & NA & NA & 9.6 \\
\hline 2003 & 16.911 .481 & 1,8 & 1.971 .397 & 368.427 & 23 & 11,74 \\
\hline 2004 & 17.272 .043 & 2,1 & 2.329 .783 & 358.386 & 18,2 & 13,5 \\
\hline 2005 & 17.487 .481 & 1,2 & 3.180 .050 & 850.267 & 36,5 & 18,2 \\
\hline 2006 & 17.758 .872 & 1,6 & 3.488 .381 & 308.331 & 9,7 & 19,6 \\
\hline 2007 & 18.248 .133 & 2,8 & 3.938 .111 & 449.730 & 12,9 & 21,6 \\
\hline 2008 & 19.102 .811 & 4,7 & 4.606 .353 & 668.242 & 13,9 & 24,1 \\
\hline 2009 & 20.427 .711 & 6,9 & 5.579 .022 & 972.669 & 21,1 & 27,3 \\
\hline 2010 & 21.016 .126 & 2,9 & 6.142 .280 & 563.258 & 10,1 & 29,2 \\
\hline 2011 & 20.994 .113 & $-0,1$ & 6.714 .792 & 572.512 & 9,3 & 32 \\
\hline 2012 & 21.253 .086 & 1,2 & 7.126 .549 & 411.757 & 6,1 & 33,5 \\
\hline
\end{tabular}

Fuente: ALLEN y SEAMAN, 2014, p. 15.

Actualmente los programas de educación a distancia están presentes en el $90 \%$ de las instituciones de educación superior. A continuación presentamos algunos hallazgos del informe más reciente que hizo el Grupo Babson en colaboración con el College Board y datos adicionales del Sistema Integrado Postsecundario de Datos Postsecundarios (IPEDS por sus siglas en inglés) (ALLEN y SEAMAN, 2015).

\subsection{1. ¿Es estratégico el aprendizaje en línea?}


La proporción de líderes académicos que informan que el aprendizaje en línea es esencial para la estrategia a largo plazo de su institución ha crecido de 48,8\% en 2002 a 70,8\% en 2014 (ALLEN y SEAMAN, 2015, p. 4).

\subsection{2. ¿Cómo comparan los resultados de la oferta en línea a la presencial?}

El porcentaje de líderes académicos que califica los resultados de aprendizaje en la educación en línea como igual o superior a los presenciales creció de 57,2\% en 2003 a 77\% en 2012. La tendencia ascendente invirtió en 2013, bajando a 74,1\%, una tasa que se ha mantenido constante para el año 2014. Menos líderes califican los resultados de aprendizaje en cursos en línea como "superior" o "algo superior" al aprendizaje presencial (20.0\% a $16,3 \%$ ), pero un mayor número los califica como "iguales" (54,1\% a 57,9\%) (ALLEN y SEAMAN, 2015, p. 5).

\subsection{3. ¿Cuántos estudiantes aprenden en línea?}

Las inscripciones en línea han aumentado a tasas superiores a los de la educación superior general. El patrón, sin embargo, ha sido de disminuir las tasas de crecimiento en el tiempo. Este año marca el primer uso de datos IPEDS examinar esta tendencia (ALLEN y SEAMAN, 2015, p. 5). IPEDS recopila la información de cada institución técnica y profesional que participa en los programas de ayuda financiera estudiantil por parte del gobierno federal. Debido a las diferencias de definiciones y la tendencia de los encuestados en el Grupo Babson de sobrestimar los porcentajes de participantes en aprendizaje en línea, los porcentajes citados en el IPEDS tienden a adherir a una métrica diferente que los porcentajes basados en las encuestas del Grupo Babson. De manera que los números de estudiantes que cita IPEDS tienden a ser menos que los estimados publicados por el Grupo Babson.

\subsection{4. ¿Cuál fue la tasa de crecimiento en 2013-14?}

La tasa de crecimiento observado en 2014 por IPEDS del número de estudiantes que toman al menos un curso a distancia fue 3,7\% más baja que las tasas de crecimiento en línea anterior (basadas en las encuestas nacionales del Grupo Babson) pero aún más alta que el aumento de la matrícula de educación superior general (ALLEN y SEAMAN, 2015, p. 5). La tasa de crecimiento en la distancia de las inscripciones era muy desigual; las instituciones de cuatro años con fines de lucro registraron la primera reducción (de 66.600 o 8,7\%) (ALLEN y SEAMAN, 2015, p. 5). Las dos clases de instituciones que mostraron el mayor crecimiento son las instituciones públicas de cuatro años (aumentadas 126,82 o $7,2 \%$ ) y las instituciones sin fines de lucro privadas de cuatro años (hasta por $86.811 \mathrm{o}$ 12,7\%) (ALLEN y SEAMAN, 2015, p. 5). 


\subsection{5. ¿Cuáles son las barreras para los profesores?}

Sólo el $28 \%$ de jefes académicos dicen que sus profesores aceptan "el valor y la legitimidad de la educación en línea", una tasa substancialmente igual a la presentada en 2003 (ALLEN y SEAMAN, 2015, p. 6). La mayoría de los líderes académicos $(68,3 \%)$ siguen creyendo que "los estudiantes necesitan más disciplina para tener éxito en un curso en línea que en un curso presencial" (ALLEN y SEAMAN, 2015, p. 6).

Un número creciente de líderes académicos cree que la retención de los estudiantes es un problema mayor para los cursos en línea que para cursos presenciales (44,6\% en 2014 frente a 40,6\% en 2013, 28,4\% en 2009 y 27,2\% en 2004) (ALLEN y SEAMAN, 2015, p. 6). El esfuerzo adicional necesario para ofrecer un curso en línea, representa una barrera para la instrucción en línea 78.0\% de líderes académicos (Allen y Seaman, 2015, p. 6).

\subsection{6. ¿Cuál es la situación actual de los MOOCs?}

El informe de Allen y Seaman (2015) señaló que el número de instituciones que ofrecían MOOCs había incrementado desde 2,6\% in 2012 a 5\% en 2013 y a 8\% en 2014. En cuanto a sus planes de ofrecer MOOCs, 39.9\% todavía estaban indecisos y 46,5\% reportaron que no tenían ningún plan (ALLEN y SEAMAN, 2015, p. 6). El porcentaje de instituciones que creían que los MOOCs representaban un método sostenible de ofrecer cursos en línea bajó desde 28,3\% en 2012 a 16,3\% en 2014 (SEAMAN y ALLEN, 2015, p. $6)$.

\subsection{Asociaciones profesionales relacionadas con la Educación a Distancia en Estados Unidos}

Una de las características que señala la madurez de una profesión es la formación de asociaciones profesionales que sirven para orientar, capacitar, y abogar por los intereses de personas que se reconoce que están involucrados mutuamente en la misma línea de trabajo. Para los que trabajan en las actividades de diseño, oferta y evaluación de programas de la educación a distancia, ésta es una lista (OLGREN, 2012a) de algunas asociaciones profesionales que se enfocan en varios aspectos de la educación a distancia, que les interesan a los que diseñan, enseñan, evalúan, gestionan, teorizan o investigan estos fenómenos de la educación a distancia en general o el aprendizaje en línea en particular:

- American Distance Education Consortium (ADEC).

- Association for Distance Education and Independent Learning (ADEIL).

- Association for Educational Communications and Technology (AECT).

- Consortium of College and University Media Centers (CCUMC).

- Distance Education Training Council (DETC). 
- EDUCAUSE.

- eLearning Guild.

- Instructional Telecommunications Council (ITC).

- Interactive Multimedia and Collaborative Communications Alliance (IMCCA).

- International Association for K-12 Online Learning (iNACOL).

- International Society for Performance Improvement (ISPI).

- International Society for Technology in Education (ISTE).

- Sloan Consortium (Sloan-C).

- Society for Applied Learning Technology.

- U.S. Distance Learning.

- Association (USDLA).

\section{$\underline{\text { 3.5. Publicaciones estadounidenses relacionadas con la Educación a Distancia }}$}

Otra característica que señala la madurez de una profesión es el estado de las publicaciones que refuerzan tales aspectos como la teoría, la investigación, la gestión y la práctica informada de sus socios. Mientras existen revistas cuyo acceso es limitado a los que pagan, otras revistas comprenden parte del movimiento para el acceso abierto. A continuamos presentamos los dos tipos de revistas (OLGREN, 2012b).

\section{$\underline{3.5 .1 . ~ R e v i s t a s ~ d e ~ p a g o ~}$}

Existen revistas que abordan temas relacionados con diferentes aspectos de la educación a distancia. Éstas son editadas (por medio de la imprenta o por el Web) por asociaciones profesionales y facilitadas a sus socios cuyas suscripciones a menudo se incluyen con la cuota de membresía. También hay editoriales comerciales que difunden información con fines de lucro que ponen en venta suscripciones y artículos individuales.

- American Journal of Distance Education: US\$99.

- CITE Journal: Contemporary Issues in Technology and Teacher Education: Suscripción incluida en la cuota de socio de la Association for the Advancement of Computing in Education (Asociación para el Avance de Computación en Educación)

- Journal of Asynchronous Learninng Networks: Suscripción incluida en la cuota de socio del Online Learning Consortium (Consorcio de Aprendizaje en Línea).

- Journal of Instruction Delivery Systems (JIDS): Suscripción incluida en la cuota de socio de la Society for Applied Learning Technology (Sociedad de Technology de Aprendizaje Aplicada).

- Journal of Research on Technology in Education (JRTE): Suscripción incluida en la cuota de socio de la International Society for Technology in Education (Sociedad Internacional para la Tecnología en Educación. 


\section{$\underline{\text { 3.5.2 Revistas de acceso abierto }}$}

En contraste con las revistas de fuentes cerradas de información, estas publicaciones se adhieren al movimiento de acceso abierto y libre a la información que proveen en sus artículos sobre múltiples aspectos de la educación a distancia.

- Educational Technology y Society.

- EDUCAUSE Quarterly Archives.

- EDUCAUSE Review.

- eLearning Magazine.

- eSchool News Online.

- Faculty Focus.

- Interdisciplinary Journal of E-Learning and Learning Objects (iJELLO).

- International Journal of Instructional Technology y Distance Learning.

- International Journal of Interactive Mobile Technologies (iJIM).

- International Journal on E-learning.

- Journal of Educators Online (JEO).

- Journal of Interactive Online Learning.

- Language Learning y Technology.

- Learning Solutions Magazine.

- Internet Learning.

- Journal of Computer-Mediated Communication.

- MERLOT Journal of Online Learning and Teaching.

- Online Journal of Distance Learning Administration.

- TechTrends.

- T.H.E. Journal.

- Usability News.

\subsection{Programas de Postgrado en disciplinas relacionadas con la Educación a Distancia}

Los que trabajan en programas de educación a distancia saben que existen distintos itinerarios formativos para diseñar, desarrollar, evaluar y gestionar cursos a distancia. Muchos aprenden sus competencias de forma experiencial al ocupar múltiples puestos y/o actividades relacionadas con la utilización de tecnologías. Para ayudarles a formar y/o reforzar su conocimiento y habilidades con respecto a diferentes aspectos de la educación a distancia, tienen la opción de cursar relevantes programas de postgrado. Estos incluyen aspectos de la tecnología, administración de la educación superior, y diseño instruccional. No existe un itinerario único. Sin embargo, reflejando la diversidad de competencias necesarias, hay una gran cantidad de programas que permiten que los estudiantes se especialicen en una carrera en educación a distancia. Por ejemplo, encontramos 642 universidades que ofrecen estudios de postgrado en tecnología educativa, de entre estos, 27 
ofrecen la especialización en diseño instruccional y 5 disponen de estudios de postgrado en educación a distancia/diseño de e-aprendizaje (e-learning).

\subsection{La Cuestión de la calidad}

\subsection{1. ¿Cómo se compara la educación a distancia con la educación presencial?}

Aunque la generación actual de la enseñanza en línea ha avanzado más allá de la generación de la educación por correspondencia que, debido a engañosas prácticas de algunas instituciones privadas (EDELSON y PITMAN, 2001), habían incurrido dudas sobre la calidad de sus cursos, el tema sigue siendo una cuestión vigente. Se notó que la cuestión de calidad de la enseñanza en línea subyacía todos los estudios que realizó el Grupo Babson. Desde su primer informe (2003), Allen y Seaman preguntaron a los oficiales académicos cómo comparaban los resultados de la enseñanza en línea con los de la enseñanza presencial. Reportaron que eran "inferior" (10.7\%), "algo inferior" (11.7\%), "igual" (44.9\%), "algo superior" (11.7\%) y "superior" (6\%). En su informe más reciente, (ALLEN y SEAMAN, 2015, p. 18) los oficiales académicos reportaron que eran "inferior" (1.4\%), "algo inferior" (9.2\%), "igual" 56.6\%, "algo superior" (25.0\%) y "superior" (7.9\%). Aunque la mayoría (89.5\%) de los oficiales académicos en este estudio más reciente percibieron el aprendizaje en línea como algo igual o superior que la educación presencial, el hecho que todavía uno de cada cinco encuestados mantenía la percepción que en el aprendizaje en línea faltaba calidad, sugiere la idea de que posiblemente esta percepción sea compartida por otros en la sociedad y recalca la importancia que las instituciones que proveen la educación a distancia constantemente validen y comuniquen abiertamente la calidad de sus cursos y programas en línea.

\subsubsection{Calidad y las asociaciones acreedoras}

Esta cuestión de la calidad de la educación a distancia es, a la vez, una preocupación prioritaria también para el gobierno federal y los gobiernos de los estados. De hecho, insisten en que los cursos y programas a distancia cumplan con criterios de calidad como una precondición para concederles a los estudiantes apoyo financiero (subvenciones y garantías para préstamos gubernamentales y privados). Además de responsabilizar a las mismas instituciones proveedoras, los gobiernos han delegado a las asociaciones de acreditación la tarea de acreditar las instituciones que cumplen con las normas establecidas de calidad de enseñanza a distancia. Hay seis asociaciones regionales y dos asociaciones nacionales que acreditan la calidad de la educación superior en los Estados Unidos. Además de los estándares que norman para todos los aspectos generales de las instituciones de educación superior, éstas han colaborado con los oficiales de las distintas instituciones en la fijación de estándares interregionales que abarcan los programas de educación a distancia (MIDDLE STATES COMMISSION ON HIGHER EDUCATION, 2011). 
A continuación presentamos "Los Nueve Sellos de Calidad" que cualquier institución ha de cumplir si busca obtener que la Middle States Commission on Higher Educación (2011, p. 3) apruebe sus cursos y programas de educación a distancia (MIDDLE STATES COMMISSION ON HIGHER EDUCACIÓN, 2011: 3):

- El aprendizaje en línea corresponde a la misión y propósito de la institución

- Los planes de la institución para desarrollar, sostener y, si apropiado, expandir la oferta en línea, están integrados a los procesos regulares de planeación y evaluación.

- El aprendizaje en línea se incorpora en los sistemas de gobernación y gestión académica de la institución.

- Los planes de estudio para la oferta en línea son coherentes, cohesivos y comparables en rigor académico a los programas ofrecidos en los formatos de instrucción tradicionales.

- La institución evalúa la eficacia de su oferta en línea, en la medida en que logre los objetivos y utilice los resultados de sus evaluaciones para alcanzar los objetivos.

- Los profesores responsables de los programas de aprendizaje en línea y la evaluación del éxito de sus estudiantes en el logro de los objetivos de aprendizaje en línea están debidamente calificados y son apoyados de forma eficaz.

- La institución provee servicios estudiantiles y académicos eficaces para apoyar a los estudiantes inscritos en los cursos y programas de aprendizaje en línea.

- La institución provee recursos suficientes para apoyar y, si es apropiado, ampliar la oferta de aprendizaje en línea.

- La institución asegura la integridad de su oferta de aprendizaje en línea.

\subsubsection{Asuntos de calidad}

Además de la influencia de las instituciones mismas y las asociaciones de acreditación regionales, existe una organización sin fines de lucro llamada Asuntos de Calidad (QM por sus siglas en inglés: Quality Matters) que ha colaborado con los esfuerzos relacionados con la educación a distancia al nivel de las instituciones. Trabaja con las instituciones y personal de las escuelas de educación primaria, secundaria y postsecundaria para diseminar información y capacitación con respecto a las acciones de establecer, monitorear y comprobar sus programas a distancia. En su página Web esta organización, Quality Matters (QM, 2014a) (Asuntos de Calidad), explica su misión:

QM es un proceso de revisión por pares centrada en el profesorado, que está diseñado para certificar la calidad de los cursos en línea y semipresenciales. QM es un líder en el aseguramiento de la calidad de la educación en línea y ha recibido reconocimiento nacional por su enfoque escalable basada en pares y la continua en la educación en línea y aprendizaje de los estudiantes. 
QM (2014b) ha creado una "Rúbrica de Calidad" que provee una lista de control para estructurar una evaluación. Abarca objetivos, medidas de evaluación, construcción de materiales, actividades de interacción, tecnología del curso, apoyo estudiantil, y accesibilidad y usabilidad. Ofrece talleres de capacitación en situ y por web a una audiencia amplia compuesta de diseñadores de instrucción, profesores, instructores, maestros y administradores de la educación primaria, secundaria y superior en el uso de herramientas para facilitar la evaluación. QM ofrece capacitación in situ y en línea para certificar las competencias relacionadas con diferentes aspectos de calidad de la educación a distancia. Actualmente QM cuenta con más de 800 suscriptores institucionales. (QUALITY MATTERS, 2014a).

\subsection{La cuestión de la autorización de los estados}

Relacionado con la cuestión de calidad de los programas de la educación es el deseo por parte de los diferentes niveles de gobierno de proteger al consumidor de cursos o programas a distancia de calidad deficiente. Esta preocupación motivó en 2010 al Departamento de Educación de los Estados Unidos (USDOE por su siglas en inglés) a agregar una nueva interpretación de una cláusula de la renovada Higher Education Act of 1965 (la Ley de la Educación Superior de 1965). La propuesta regla de la educación a distancia (34 C.F.R. 600.9c) requiere que las instituciones de enseñanza superior que ofrecen programas en línea obtengan la autorización en todos los estados donde residen sus estudiantes (DEPARTMENT OF EDUCACIÓN, 2010). También exige que los estados establezcan nueva reglamentación para la educación a distancia que llega de otros estados. Estas iniciativas resultaron en una severa limitación del acceso de estudiantes a través de fronteras estatales y sobre la conciencia de las distintas instituciones proveedoras respecto a que, si no se conformaran con la autorización de los diferentes estados, sus estudiantes inscritos en tales programas perderían su elegibilidad para concesiones y préstamos del gobierno federal.

Aunque siempre existía la necesidad de las instituciones de la educación superior de cumplir con las reglas de los estados en los cuales operaba programas a distancia, no todas las instituciones prestaban el mismo grado de atención a esta necesidad. Con los anuncios de la intención del gobierno federal de eliminar su ayuda financiera a los estudiantes de instituciones recusantes, las instituciones se dieron cuenta de los gastos potencialmente altos de cumplir con la reglamentación estatal. Para evitar tales cuotas algunas universidades ya rehúsan inscripciones de estudiantes de otros estados. Otras instituciones aceptan los costos financieros y administrativos de someter a los diferentes estados los informes y peticiones anuales exigidas para ofrecer sus programas legítimos en cada estado donde haya estudiantes inscritos. 
El Council for Higher Education Accreditation (CHEA por sus siglas en inglés, Consejo de Acreditación de Educación Superior) ha descrito la naturaleza de esta situación disfuncional así:

Los estados regulan la oferta de educación postsecundaria dentro de sus fronteras con diferentes estándares, políticas, prácticas, y "disparadores" para la aplicación y el cumplimiento. Las instituciones varían en el grado que ponen atención a la regulación de los estados, particularmente con respecto a las actividades que persiguen fuera de su estado natal y especialmente con respecto a los estudiantes "en línea" o "a distancia". Actualmente no existe alternativa a la necesidad de que cada institución por separado persiga autorización estatal (o garantía que no se requiera autorización) en cada estado y territorio en el cual sirve a estudiantes. Por consiguiente, miles de instituciones deben buscar aprobación y autorización de hasta 54 Estados y territorios. Ese enfoque es ineficiente y un reto para las instituciones y los Estados por igual (2012).

A pesar de esta evaluación de las implicaciones de esta cuestión de autorización estatal para la educación superior a distancia en los estados, la Comisión Interestatal del Occidente para la Educación Superior (WICHE por sus siglas en inglés), una organización regional compuesta de 16 estados del oeste de los Estados Unidos, ha intentado a moderar el debate con esta explicación:

Sólo unos pocos de los estados requieren autorización si la única actividad que emprende una institución es tener en línea estudiantes que residen en los estados. Algunos estados exigen que una institución solicite una exención, mientras que otros no requieren que las instituciones hagan nada en absoluto (WICHE, 2014).

Con el propósito de ayudar a transformar la entrega de educación a distancia, un grupo diverso de educación superior y jefes de estado, acreedores y reguladores liderado por el ex Secretario de Educación de los Estados Unidos Richard Riley, reveló planes para un nuevo sistema interestatal de reciprocidad que simplificará significativamente las regulaciones actuales y permitirá que las universidades y colegios ofrezcan más fácilmente cursos en línea en todo el país. La Comisión sobre el Informe del Reglamento de Educación Postsecundaria a Distancia busca liberar a las instituciones de educación superior del laberinto de regulaciones inconsistentes, ineficientes y costosas, además de liberarlos de leyes en diferentes estados que a menudo hacen difícil ofrecer cursos en línea a los estudiantes que residen fuera de estado donde opera la institución. El plan también atiende la protección del consumidor para proteger a los estudiantes (STATE HIGHER EDUCACIÓN EXECUTIVE OFFICERS ASSOCIATION, 2013).

Para aliviar la carga reglamentaria de las instituciones de educación superior que ofrecen educación a distancia, y a la vez hacer más factible para las oficinas rectoras de la educación superior en los estados a confeccionar sus políticas conformes con la disposición del USDOE, las asociaciones acreditadoras regionales han colaborado en la formación de un Consejo Nacional del Acuerdo de Autorización Reciproca de los Estados (SARA por 
sus siglas en inglés: el National Council for State Authorization Reciprocity Agreements), el cual es financiado por la Fundación Lumina y por las cuotas de las distintas universidades, el propósito de este acuerdo es unir los esfuerzos de los estados para establecer reglamentos nacionales comparables y facilitar la entrega de educación a distancia que origina en otros estados. Los acuerdos de reciprocidad entre los estados que contempla SARA facilitará la aprobación en varios estados a la vez (STRATFORD, 2014). En diciembre de 2014, ya se habían unido más de un tercio de los estados a SARA. Muchos esfuerzos estuvieron en proceso para aprobar legislación en otros estados, lo que les permitirá a unirse a las actividades asociadas con SARA (HORN, 2013). El Department of Educación (DSOE) ha fijado varias fechas para la implementación de sus reglamentos concernientes a la autorización de los estados, pero debido a la resistencia de las instituciones, ha habido varias demoras. En junio de 2014, el DSOE anunció otra fecha límite para el $1^{\circ}$ de julio del 2015. Se espera que asuma una posición de flexibilidad hacia estos arreglos que tienen como fin ayudar a las instituciones proveedoras de educación en línea como las unidades de los estados encargadas de la entrega de la educación a distancia provenientes de otros estados.

\section{COMPARACIÓN DE LA EDUCACIÓN A DISTANCIA EN AMBOS PAÍSES}

Para comparar la evolución e implicaciones de la educación a distancia en México y en Estados Unidos de América, hemos considerado pertinente tomar como eje central algunos aspectos del análisis anteriormente presentado, mismos que se discuten a continuación.

\subsection{Origen}

Podemos encontrar diferencias interesantes en el origen de la educación a distancia en ambos países. Mientras que en Estados Unidos surge como una iniciativa privada para fomentar los estudios en casa y posteriormente logra el reconocimiento oficial y se le permite el otorgamiento de grados, en México la primera iniciativa registrada surge de instancias gubernamentales con el fin de subsanar una necesidad en el campo educativo, es decir, desde sus inicios era oficialmente reconocida. En el primer caso es la sociedad quien demanda el establecimiento y reconocimiento de los estudios a distancia, lo que implica un nivel de compromiso con la modalidad por parte de los directamente implicados, mientras que en el segundo es una iniciativa gubernamental enfocada a un grupo social específico, muy probablemente sin consultar o socializar previamente la idea. En los dos casos las diferencias de origen pueden tener implicaciones directamente proporcionales a la aceptación social de la educación a distancia.

\subsection{Las generaciones}


En este punto consideramos que no hay diferencias sustanciales, pues las generaciones definidas para Estados Unidos aplican en el mismo sentido en México y en el resto del mundo. La primera generación se basó en impresos distribuidos por medio de correo convencional con un nivel de interacción entre el docente y el estudiante extremadamente bajo; la segunda empleó medios masivos de comunicación como radio y televisión con apoyo de impresos distribuidos por correo convencional; la tercera empleó ordenadores y software especializados, así como medios electrónicos diversos; la cuarta se distingue por el uso de Internet y finalmente la quinta sigue empleando el internet como medio de comunicación pero emplea las capacidades asociativas e interactivas del Internet en un modelo altamente interactivo no sólo entre docente y estudiante, sino con el grupo de estudio también. Desde esta perspectiva no existen diferencias significativas entre ambos países.

\subsection{La acreditación federal vs acreditación estatal}

En este punto sí existen diferencias importantes entre los dos países. Salvo en algunas profesiones, en Estados Unidos se considera pertinente la acreditación de un programa en el nivel estatal, y un egresado de un programa educativo en un estado está plenamente capacitado para trabajar profesionalmente en otro estado. En el caso de México la situación cambia, porque un programa educativo puede tener reconocimiento de validez estatal, sin embargo el egresado no queda certificado para trabajar profesionalmente en otro estado, sólo tendrá reconocimiento su grado en el estado donde haya cursado sus estudios. Por esta razón son pocas las instituciones que eligen validar sus programas estatalmente y prácticamente todos optan por tramitar la validación federal, pues entonces el egresado estará en condiciones de laborar en cualquier parte del país y del extranjero. Esta diferencia en la validación de los programas educativos ha repercutido favorablemente en México en lo relacionado con la educación a distancia pues se cuenta con el reconocimiento de validez oficial, y los mecanismos de obtención de esa validez oficial son procesos muy explorados y, aunque son complicados, son del dominio de las instituciones. En el caso de los Estados Unidos el panorama cambia y está en proceso de simplificar sus trámites para que los programas educativos a distancia puedan ser validados por organizaciones acreedores interregionales y así reconocidos de manera federal.

\subsection{El crecimiento}

En este aspecto encontramos diferencias significativas entre los dos países. La educación a distancia ha tenido mayor crecimiento en Estados Unidos y ese crecimiento ha sido constante desde sus inicios, aunque es cierto que en los últimos 20 años su crecimiento se ha acelerado considerablemente, mientras que en México el crecimiento fue muy lento en los primeros años y en los últimos 20 se ha acelerado, pasando en poco de tiempo de ser una opción de calidad dudosa a la alternativa con calidad educativa y pertinencia real. Las 
diferencias entre ambos países se deben a varias causas, entre las que podrían considerarse la cantidad y calidad de infraestructura tecnológica en telecomunicaciones, la dispersión geográfica de la población, la visión de la sociedad que en un caso estuvo directamente implicada desde los inicios y en el otro se mantuvo en una postura reservada, las diferencias en presupuesto federal asignado a la educación y, finalmente, las necesidades propias de la sociedad en cuanto a formación profesional. La demanda de la sociedad por educación en esta modalidad ha ido en aumento en ambos países, y se espera un crecimiento sostenido en los próximos años.

\subsection{Las organizaciones paralelas}

En ambos países han surgido organizaciones e instancias tanto gubernamentales como no gubernamentales que han dedicado sus esfuerzos a generar insumos para la educación a distancia como investigación especializada, lineamientos para la evaluación de la calidad de programas, estudios sobre las herramientas nuevas que posibilitan nuevos enfoques y horizontes, e incluso la formación de analistas y docentes especializados en esta modalidad educativa. De la misma manera existen empresas privadas que se dedican a proveer servicios de Internet y de software especializado para crear los ambientes de aprendizaje que se requieren. El avance en ambos países corre por la misma línea, aunque con las diferencias que implica la economía en cada sitio.

\subsection{Publicaciones}

Como indicadores de madurez en este estudio se han considerado la generación de publicaciones especializadas, la oferta de posgrados relacionados con educación a distancia y los indicadores de incremento en matrícula de los hemos hablado, a la par del surgimiento de organizaciones en pro de la educación a distancia mencionadas en el apartado anterior. Respecto a la generación de publicaciones especializadas se pueden observar diferencias importantes entre ambos países, pues en México se cuenta con pocas publicaciones especializadas propias, aunque se ha invertido en el apoyo de publicaciones sobre educación a distancia en Hispanoamérica. Sin embargo en Estados Unidos se han generado y mantenido múltiples publicaciones tanto libres como de pago, mismas que se han posicionado como espacios de calidad para compartir y socializar avances y trabajos relacionados con la modalidad. Una parte importante en este trabajo es la colaboración con interesados en la educación a distancia en todo el mundo. Respecto a la oferta de posgrados relacionados con la educación a distancia podemos encontrar equivalencias en ambos países, lo cual implica conciencia de la importancia de fundamentar adecuadamente los avances en esta modalidad mediante la formación idónea para diseñadores instruccionales, docentes, gestores, tecnólogos e investigadores.

\subsection{Calidad}


En ambos países se pueden encontrar avances importantes en cuanto a la generación de lineamientos, reglamentos y procesos para asegurar la calidad de los programas educativos que se ofrecen a distancia, a la par de que se invierte en la formación de expertos capacitados en la evaluación de programas. En el caso de Estados Unidos se busca la modificación de la ley para que los programas puedan ser validados por las asociaciones interregionales y entonces aceptados en el ámbito Federal, lo cual repercute en beneficio de quienes accedan a un programa educativo a distancia. En el caso de México se trabaja para que las instancias federales refinen sus instrumentos y esquemas que ya existen para el aseguramiento de la calidad, y por supuesto, que se homologue la posibilidad de acceso a becas de estudios para los estudiantes a distancia.

\subsection{La prospectiva}

Hacia dónde vamos en educación a distancia es un tema que despierta interés generalizado a distintos niveles de la sociedad y del gobierno tanto en México como en Estados Unidos de América. Sin lugar a dudas existe un interés en aumento por los estudios en esta modalidad, el poder franquear las barreras de tiempo y espacio es un aspecto que llama poderosamente la atención a poblaciones con tendencia al envejecimiento, lo que implica que el grueso de la población está en edad productiva y esto supone limitaciones de tiempo y espacio para atender programas educativos presenciales. Es clara la importancia que la educación a distancia representa para la sociedad. Afortunadamente los avances en el aseguramiento de la calidad ayudan a que se diluya la percepción sobre su escasa calidad.

\section{CONCLUSIONES}

La educación a distancia en México tiene muchos años ya en el panorama educativo. Desde sus inicios fue concebida como una alternativa para favorecer procesos educativos en condiciones y tiempos donde la educación presencial no tenía cabida. El proceso de crecimiento ha sido lento. Sin embargo en los últimos 20 años ha evolucionado rápidamente de forma tal que, en el momento actual, la mayoría de las instituciones de educación superior en el país han incorporado cursos o programas en esta modalidad, desde cursos únicos, hasta programas de técnico superior universitario, licenciaturas, especialidades, maestrías y doctorados.

Las instancias encargadas de validar la calidad de los programas educativos en el país han desarrollado esfuerzos importantes por diseñar instrumentos y procesos especializados en esta modalidad educativa a fin de garantizar su calidad, lo cual ha sido factor de confiabilidad tanto para la población que opte por un programa educativo a distancia como por los distintos niveles de gobierno que otorgan ayuda financiera a los estudiantes. 
Se han formado organizaciones con el fin de generar investigación que ofrezca las bases para un crecimiento en la educación a distancia ordenado y acorde a las necesidades y requisitos de la población en México. De igual forma, han surgido proyectos nacionales con el fin de atender no solamente las necesidades del país en educación superior, sino también de fomentar la colaboración con otros países y generar espacios de coincidencia y construcción común del conocimiento.

La educación a distancia en los Estados Unidos de América es un fenómeno que está en pleno crecimiento. Hemos delineado las generaciones anteriores que se utilizaban para ofrecer oportunidades de aprendizaje a personas que de otro modo habrían dificultad de acceder. Hemos identificado cuatro tipos de oferta actual de la educación a distancia que entregan las instituciones de la educación superior. Las instituciones que ofrecen los primeros dos tipos se distinguen por su misión de servir a estudiantes exclusivamente a distancia. El primer tipo se enfoca en reconocer y validar competencias en vez de obtener créditos por cursos completados. El segundo tipo ofrece cursos y programas exclusivamente a distancia. El tercer tipo ofrece cursos y programas por los dos modos: presencialmente en aulas ubicadas en recintos de "ladrillos y mortero" y en línea a través de Internet. El cuarto tipo comprenden los MOOCs, un tipo innovador de entrega gratuita que alcanza hasta miles de estudiantes. Con base en encuestas y estudios nacionales hemos notado atributos de la educación a distancia que se encuentran en los sistemas educativos de las escuelas primarias, secundarias e instituciones postsecundarias. Hemos notado también varias muestras de la madurez de la educación a distancia como un campo de práctica y estudio, tales como las asociaciones y revistas profesionales así como los programas de estudios postgrados en disciplinas relacionadas con la educación a distancia. Aunque no se han publicado cifras sobre su extensión actual, en vista de los patrones de las tasas de crecimiento en todos los sistemas educativos, nos quedamos con la confianza que las inscripciones en programas a distancia seguirán en ascenso en el futuro previsible. La educación a distancia ya se ha integrado en todos los sistemas educativos.

\section{REFERENCIAS BIBLIOGRÁFICAS}

ALLEN, I. E. y SEAMAN, J. (2004): Entering the Mainstream: The Quality and Extent of Online Education in the United States, 2003 and 2004: Overview. Extraído de http://www.onlinelearningsurvey.com/reports/entering-the-mainstream.pdf

ALLEN, I. E. y SEAMAN, J. (2003): Sizing the Opportunity: The Quality and Extent of Online Education in the United States, 2002 and 2003. The Sloan Consortium. Extraído de file:///C:/Users/Peter/Downloads/sizing_opportunity_2\%20(3).pdf

ALLEN, I. E. y SEAMAN, J. (2005): Growing by Degrees: Online Education in the United States, 2005 (Needham, MA, Sloano-C). 
ALLEN, I. E. y SEAMAN, J. (2006): Making the Grade: Online Education in the United States (Needham, MA, Sloano-C).

ALLEN, I. E. y SEAMAN, J. (2007): Online Nation: Five Years of Growth in Online Learning (Needham, MA, Sloan-C).

ALLEN, I. E. y SEAMAN, J. (2008): Staying the Course: Online Education in the United States, 2008 (Needham, MA: Sloan-C).

ALLEN, I. E. y SEAMAN, J. (2009): Learning on Demand: Online Education in the United States, 2009 (Needham, MA: Sloan-C).

ALLEN, I. E. y SEAMAN, J. (2010): Class Differences in the United States: Online Education in the United States, 2010 (Needham, MA: Sloan-C).

ALLEN, I. E. y SEAMAN, J. (2011): Going the Distance: Online Education in the United States 2011 (Needham, MA, Sloan-C).

ALLEN, I. E. y SEAMAN, J. (2012): Growing the Curriculum: Open Education Resources in U.S. Higher Education (Needham, MA: Babson Survey Research Group).

ALLEN, I. E. y SEAMAN, J. (2013): Changing course: Ten Years of Tracking Onlne Edcation in the United States (Needham, Babson Survey Research Group). Extraído de http://www.onlinelearningsurvey.com/reports/changingcourse.pdf

ALLEN, I. E. y SEAMAN, J. (2015): Grade level: Tracking online education in the United States. With commenetary about IPEDS as the new data source. Babson Survey Research Group and Quahog. Extraído de http://onlinelearningconsortium.org/read/survey-reports-2014/

ALLEN, I. E. y SEAMAN, J. (2014): Opening the Curriculum: Open Educational Resources in U.S. Higher Education, 2014. Babson Survey Research Group. Extraído de http://onlinelearningsurvey.com/reports/openingthecurriculum2014.pdf

ALTILLO.COM (2015): Universidades Virtuales en México. El Portal de los Estudiantes. Extraído de http://www.altillo.com/universidades/universidades_mexol.asp

ANUIES (2015): Información Estadística de Educación Superior. Extraído de http://www.anuies.mx/content.php?varSectionID=142

BENKE, M., DAVIS, A. y TRAVERS, N. L. (2012): SUNY Empire State College: A name changer in open learning, en D. G. OBLINGER (Ed.). Game changers: Education and Information Technologies, pp. 144-157 (EDUCAUSE).

BOSCO, H. D. (2015): La Educación A Distancia en México: Narrativa de una Historias Silenciosa (México DF, Universidad Nacional Autónoma de México, México).

CENTRO DE ACTUALIZACIÓN DEL MAGISTERIO (2015). Antecedentes. Extraído de http://cam.tamaulipas.gob.mx/antecedentes.

CHEA (2012): "SARA" State Authorization Reciprocity Agreement Presentation: A nationwide process to make state authorization more uniform, effective and efficient. State Authorization Reciprocity Agreement Presentation CHEA 2012. Extraído 
http://www.chea.org/powerpoints/Revised\%20SARA\%20Presentation\%20June \%20 4\%202012.pdf (Consultado el 10 de marzo de 2015).

CLARK, J. J. (1906): The correspondence school. Its relation to technical education and some of its results, Science, 24(611), 327-334.

COLLEGE BOARD (2015): Trends in College Pricing. Extraído de http://trends.collegeboard.org/college-pricing/figures-tables/average-rates-growthpublished-charges-decade

COUNCIL FOR HIGHER EDUCATION ACCREDITATION (CHEA) (2012): State Authorization Reciprocity Agreement: A nationwide process to make state authorization more uniform, effective and efficient. Extraído de http://www.chea.org/powerpoints/Revised\%20SARA\%20Presentation\%20June\%20 4\%202012.pdf

DANIEL, S. J. (2012): Making Sense of MOOCs: Musings in Maze of Myth, Paradox and Possibility. Journal of Interative Media in Education, 3(18). doi: 10.5334/2012-18

DEPARTMENT OF EDUCATION (2010): Program Integrity Issues. State Authorization. Federal Register, 75(209), pp. 66858-66867.

EDELSON, P. J. y PITMAN, V. V. (2001): C. Global E-Journal of Oped, Flexible and Distance Education, 1, pp. 71-83.

GAMBOA HERRERA, J. I. (2014): Las Misiones Culturales entre 1922 y 1927. Extraído de

http://www.comie.org.mx/congreso/memoriaelectronica/v09/ponencias/at09/PRE11 78909741.pdf

GÓMEZ QUINTERO, N. (2012): En educación a distancia, lento avance: especialistas. $E l$ Universal Nación. Extraído de http://www.eluniversal.com.mx/nacion/202522.html

HORN, M. B. (2013): Digital roundup: States legislatures scramble to boost, or in some cases block, online learning. EducationNext, 13(4). Extraído de http://educationnext.org/digital-roundup/

INSTITUTO CONSORCIO CLAVIJERO (2015): Instituto Consorcio Clavijero. Extraído de http://www.clavijero.edu.mx/

LUJÁN MORA, S. (2013). MOOC (Massive Open Online Course). Extraído de http://desarrolloweb.dlsi.ua.es/moocs/

MENDENHALLl, R. W. (2012): Western Governors University, en D. G. OBLINGER (Ed.) Game Changers: Education and Information Technologies, pp. 115-132 (EDUCAUSE).

MIDDLE STATES COMMISSION ON HIGHER EDUCATION (2011): Distance education progdams: Interregional guidelines for the evaluation of distance education (online learning). Extraído de http://www.msche.org/publications/Guidelines-for-the-Evaluation-of-DistanceEducation-Programs.pdf 
MOORE, M. G. y KEARSLEY, G. (2005): Distance education: A systems vieew ( $^{\text {nd }}$ edition) (Belmont, CA, Wadsworth).

MORENO CASTAÑEDA, M. (2010): La Educación a Distancia en las IES Públicas Mexicanas. (Texto preparado para el proyecto de educación a distancia de ANUIES).

NASSEH, B. (1997): A brief history of distance education. Adult Education in the News. Extraído de http://www.seniornet.org/edu/art/history.html

OBLINGER, D. G. (2014): Where quality assurance and information technology meet. CHEA 2014 Annual Conference Presentation. CHEA. Extraído de http://www.chea.org/pdf/2014_Presentations/Oblinger\%20PowerPoint\%20Presenta tion.pdf

OBLINGER, D. G. (Ed.) (2012): Game changes: Education and information technologies. EDUCAUSE. Extraído de http://www.educause.edu/researchpublications/books/game-changers-education-and-information-technologies

OLGREN, C. H. (2012a): Associations y Professional Organizations. University of Wisconsin-Madison Distance Education Certificate Program.

OLGREN, C. H. (2012b): Journals y Magazines. University of Wisconsin-Madison Distance Education Certificate Program.

PICCIANO, A. G. (2007): Blended Learning: Research Perspectives (Needham, MA, Sloan Center for OnLine Education).

PITTMAN, V. V. (2003): Correspondence study in the American university: A second Historiographic perspective, en M. G. MOORE y W. G. ANDERSON, Handbook of distance education, pp. 21-36 (Mahwah, Lawrence Erlbaum Associates, Inc., Publishers).

QUALITY MATTERS (2014a): Quality Matters. About QM. Extraído de https://www.qualitymatters.org/welcome

QUALITY MATTERS (2014b): Higher Education Rubric (Quality Matters Higher Education Program). Extraído de https://www.qualitymatters.org/rubric

SABA, F. (2011): Distance education in the United States: Past, present, future, Educational Technology, 51(6), pp. 11-18.

SAETTLER, P. (1990): The evolution of American educational technology (Englewood, CO, Libraries Unlimited).

SÁNCHEZ SOLER, M. D. (1998): La Educación a Distancia en México y Propuestas para su Desarrollo. VII Encuentro Inernacional de educación a distancia en la feria Internacional del Libro fIL 98 (Guadalajara, Universidad de Guadalajara).

STATE HIGHER EDUCATION EXECUTIVE OFFICERS ASSOCIATION (SHEEO) (2013): Final Report of the Distance Education commission: Advancing Access through Regulatory Reform. Extraído de http://www.sheeo.org/resources/publications/final-report-distance-educationcommission-advancing-access-through 
WATKINS, J. D. (1996): Education for success: The International Correspondence Schools of Scranton, Pennsylvania, Pennsylvania Magazine of History and Biography, pp. 343-369.

WICHE Cooperative for Educational Technologies (2014): State Authorization and MOOCs. Extraído de http://wcet.wiche.edu/wcet/docs/talkingpoints/WCETTalkingPoings-StateAuthorizationAndMOOCs-04-17-2014.pdF

\section{PROFESIOGRAFÍA}

\section{Pedro Cookson Steele}

Consultor con la empresa educativa Adelante Online. Anteriormente desempeñaba funciones de director de cursos en línea en una universidad pública del estado de Delaware, Estados Unidos, director académico del Instituto Consorcio Clavijero en Xalapa, Veracruz, México y vice-presidente académico asociado con la Universidad de Athabasca, Alberta, Canadá. Ha sido editor fundador de la revista International Review of Research in Open and Distance Educación (www.irrodl.org).

Datos de contacto: Adelante Online, 96 Beacon Hill Drive, Dover, Delaware 19904. Email: pedroscookson@ outlook.com / peterscookson@gmail.com

\section{Nancy Domínguez González}

Docente en la Universidad Veracruzana donde coordina el Laboratorio Multimedia de la Desarrollo Biolögico Agropecuaria Xalapa y en la Universidad DAVici es diseñadora instruccional, docente y fue coordinadora de la Maestría en Tecnología Educativa Virtual. Desarrolla investigación en educación a distancia, educación en línea y sobre procesos de incorporación de TICSs en la docencia, la investigación y la vinculación.

Datos de contacto: Universidad Veracruzana, Lomas del Estadio S/N, Facultad de Ciencias Agrícolas, Xalapa, 91000.

Fecha de recepción: 30 de mayo de 2015 .

Fecha de aceptación: 28 de diciembre de 2015. 